\title{
Neutron measurements at the ELISE neutral beam test facility and implications for neutron based diagnostics at SPIDER
}

S. Feng, M. Nocente, D. Wünderlich, F. Bonomo, G. Croci, U. Fantz, B. Heinemann, W. Kraus, I. Mario, A. Muraro, R. Pasqualotto, M. Rebai, M. Tardocchi, and G. Gorini

Citation: Review of Scientific Instruments 89, 101139 (2018); doi: 10.1063/1.5036823

View online: https://doi.org/10.1063/1.5036823

View Table of Contents: http://aip.scitation.org/toc/rsi/89/10

Published by the American Institute of Physics

\section{Articles you may be interested in}

Design of gamma-ray spectrometers optimized for fast particle studies at ITER

Review of Scientific Instruments 89, 101126 (2018); 10.1063/1.5038963

Calibration of a neutron time-of-flight detector with a rapid instrument response function for measurements of bulk fluid motion on OMEGA

Review of Scientific Instruments 89, 101131 (2018); 10.1063/1.5037324

Measurement of apparent ion temperature using the magnetic recoil spectrometer at the OMEGA laser facility

Review of Scientific Instruments 89, 101129 (2018); 10.1063/1.5035287

Development of an ultra-fast photomultiplier tube for gamma-ray Cherenkov detectors at the National Ignition Facility (PD-PMT)

Review of Scientific Instruments 89, 101137 (2018); 10.1063/1.5039327

Gallium nitride ( $\mathrm{GaN}$ ) devices as a platform technology for radiation hard inertial confinement fusion diagnostics

Review of Scientific Instruments 89, $10 \mathrm{~K} 113$ (2018); 10.1063/1.5039407

Velocity-space sensitivity of the compact neutron emission spectrometers at EAST

Review of Scientific Instruments 89, 101141 (2018); 10.1063/1.5039393

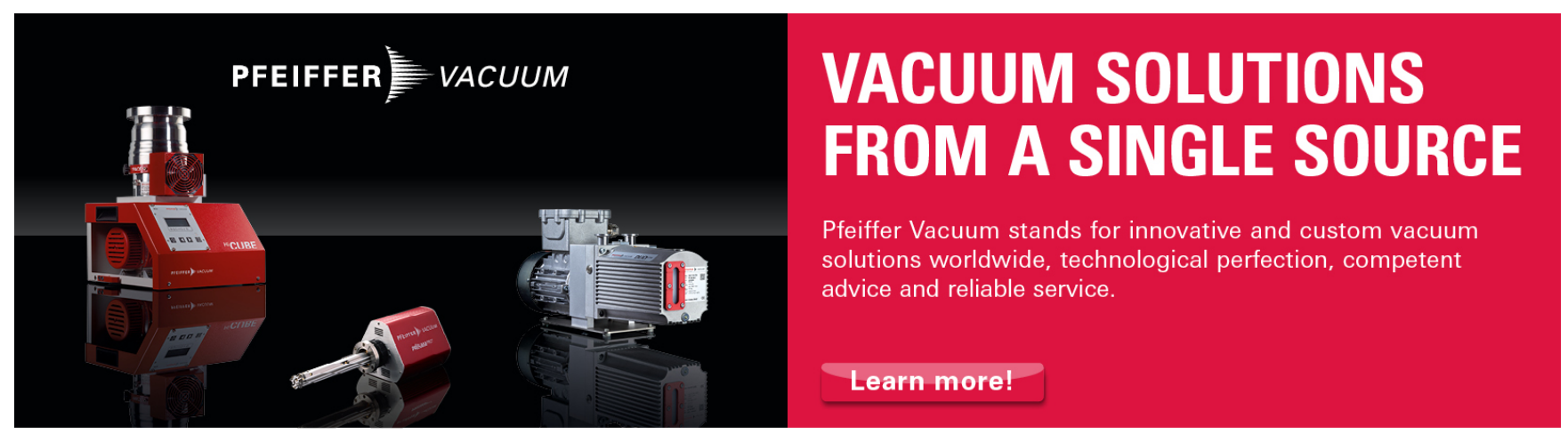




\title{
Neutron measurements at the ELISE neutral beam test facility and implications for neutron based diagnostics at SPIDER
}

\author{
S. Feng, ${ }^{1, a)}$ M. Nocente, ${ }^{1,2, b)}$ D. Wünderlich, ${ }^{3}$ F. Bonomo, ${ }^{3}$ G. Croci, ${ }^{1,2}$ U. Fantz, ${ }^{3}$ \\ B. Heinemann, ${ }^{3}$ W. Kraus, ${ }^{3}$ I. Mario, ${ }^{3}$ A. Muraro, ${ }^{2}$ R. Pasqualotto, ${ }^{4}$ \\ M. Rebai, ${ }^{2}$ M. Tardocchi, ${ }^{2}$ and G. Gorini ${ }^{1,2}$ \\ ${ }^{1}$ Dipartimento di Fisica "G. Occhialini," Università di Milano-Bicocca, Milano, Italy \\ ${ }^{2}$ Istituto di Fisica del Plasma "P. Caldirola," Consiglio Nazionale delle Ricerche, Milano, Italy \\ ${ }^{3}$ Max Planck Institut für Plasmaphysik, Garching, Germany \\ ${ }^{4}$ Consorzio RFX, Padova, Italy
}

(Presented 16 April 2018; received 19 April 2018; accepted 3 July 2018; published online 17 October 2018)

\begin{abstract}
Along the route to the development of a neutral beam injector for ITER, the Padua based Source for Production of Ion of Deuterium Extracted from Rf plasma (SPIDER) and megavolt ITER injector and concept advancement facilities will make use of neutron diagnostics to quantify the homogeneity of the neutral beam profile by measuring the map of the neutron emission from the beam dump with the close-contact neutron emission surface mapping (CNESM) system. Neutrons are here produced from beam-target reactions between the deuterium beam and the deuterons previously adsorbed in the calorimeter. In order to aid the interpretation of the diagnostic data, a dedicated experiment on neutron emission from beam-target reactions with beam parameters approaching those expected at SPIDER has been performed at the Extraction from a Large Ion Source Experiment (ELISE) neutral beam test facility. The time trace of neutron emission has been measured using a calibrated liquid scintillator detector at increasing power densities on the target. Compared to calculations based on the local mixing model, a systematic discrepancy was observed exceeding the statistical accuracy of the measurements and increasing as a linear function of the power density. The data are used to derive an empirical temperature dependent correction for applications to neutron measurements at SPIDER. Published by AIP Publishing. https://doi.org/10.1063/1.5036823
\end{abstract}

\section{INTRODUCTION}

The PRIMA (Padova Research on ITER Megavolt Accelerator) neutral beam test facility which is designed to demonstrate the feasibility of a prototype neutral beam injector for ITER is under construction in Padova, Italy. ${ }^{1,2}$ PRIMA includes the negative ion source SPIDER (Source for Production of Ion of Deuterium Extracted from Rf plasma) and the full power injector MITICA (Megavolt ITER Injector and Concept Advancement). SPIDER is the necessary step before MITICA because it has to demonstrate extraction and acceleration to $100 \mathrm{keV}$ of a large negative ion hydrogen or deuterium beam with uniform intensity and low divergence. In order to well estimate the beam uniformity and divergence, a set of diagnostics including the CNESM (close-contact neutron emission surface mapping) system have been designed. ${ }^{3}$ The CNESM system is based on Gas Electron Multiplier (GEM) detectors for neutron detection with the aim to resolve the 2D beam intensity profile in deuterium operations at SPIDER. ${ }^{4-7}$ Here, fusion reactions between beam deuterons and deuterons implanted in the copper beam dump will produce about $10^{12}$ neutrons/s spread over $1 \mathrm{~m}^{2}$. Then the footprint of the

\footnotetext{
Note: Paper published as part of the Proceedings of the 22nd Topical Conference on High-Temperature Plasma Diagnostics, San Diego, California, April 2018.

a) Electronic mail: f.song@ campus.unimib.it

b)Electronic mail: massimo.nocente@mib.infn.it
}

deuterium beam that hits the dump can be retrieved from the spatial map of the neutron emission intensity obtained by the CNESM. ${ }^{8-10}$ In SPIDER, CNESM will be benchmarked by the Short-Time Retractable Instrumented Kalorimeter Experiment (STRIKE), an inertially cooled calorimeter as the main diagnostics for the beam profile mainly based on Infrared (IR) measurements. As IR measurements cannot be used on MITICA due to engineering constraints, the experience gained at SPIDER will be instrumental to make CNESM the reference system for deuterium beam profile measurements on MITICA. ${ }^{11}$ In order to aid the detector development, neutron emission from $D(d, n)^{3} \mathrm{He}$ reactions in the beam target experiment must be investigated first.

A parasitic experiment was first performed at the Extraction from a Large Ion Source Experiment (ELISE) facility. ${ }^{12}$ The time evolution of beam-target neutron emission from the dump was measured by using a calibrated EJ301 liquid scintillator and has been compared with calculations based on the Local Mixing Model (LMM) ${ }^{13,14}$ on a relative scale. Calculations could reproduce the general qualitative features of the measured time trace of neutron emission but, at a more quantitative level, overestimated the neutron emission up to $30 \%$, which could be due to systematic uncertainties of the input data. Benefiting from later refinements of the method to analyze data from the diagnostic calorimeter, ${ }^{11}$ a moderate discrepancy up to $12 \%$ between the LMM based calculation and data in the power range $160 \mathrm{~kW}$ to $440 \mathrm{~kW}$ has been revealed 
in a new experiment. ${ }^{15}$ This, if extrapolated to SPIDER, would imply a deficit as large as $70 \%$ in full power deuterium operations. On the other hand, the power density on the dump at SPIDER will be a factor four larger than the values tested at ELISE in our previous experiment ${ }^{15}$ and a linear, empirical extrapolation of our results to SPIDER might be questionable.

To the purpose of verifying whether the deficit still extrapolates linearly toward the beam power expected at SPIDER, we have performed a dedicated experiment that explores a parameter range approaching the maximum capabilities allowed by the ELISE facility ${ }^{16}$ of the Max Planck Institut für Plasmaphysik (IPP) (Garching, Germany), where the beam power was scanned from about $200 \mathrm{~kW}$ to $950 \mathrm{~kW}$ by modifying the beam current and/or voltage in a controlled manner. Particular attention was paid to ensuring a constant beam size and profile as the power was scanned. A neutron detector was used to monitor the time trace of neutron emission and the result has been compared with LMM based calculations on a relative scale (see below). The results are also used to determine an empirical correction for CNESM applications at SPIDER.

\section{BEAM-TARGET NEUTRON EMISSION EXPERIMENT}

\section{A. Operation of pulsed deuterium beams}

ELISE is a half size ITER neutral beam injection (NBI) test facility which can produce a negative ion beam up to $60 \mathrm{keV}$ for $10 \mathrm{~s}(\mathrm{~s})$ every $3 \mathrm{~min} .{ }^{17,18}$ The experiment here described is based on 231 deuterium pulses in 3 different experimental days. For each pulse, we measured the time trace of the neutron emission as a function of time with the calibrated liquid scintillator described below. The filling pressure was set to $0.6 \mathrm{~Pa}$ and the operation parameters including the average beam currents, high voltages (HV), and power on the dump in the experiment are shown in Table I. For pulses marked by an asterisk, the power on the dump was gradually increased up to the target value. For those pulses, the starting value of the power, $\mathrm{HV}$, and current used are also indicated within parentheses. The highest beam power deposited on the dump was achieved at about $950 \mathrm{~kW}$.

A copper calorimeter (with a total surface of $1.2 \mathrm{~m} \times$ $1.2 \mathrm{~m})$ consists of $30 \times 30$ blocks $^{11}$ and is placed at a

TABLE I. Operation parameters of the deuterium beam in the neutron emission experiment.

\begin{tabular}{lccc}
\hline \hline Nr. pulses & Power $(\mathrm{kW})$ & HV $(\mathrm{keV})$ & Current (A) \\
\hline 4 & 190 & 30.1 & 6.3 \\
20 & 214 & 30.1 & 7.1 \\
22 & 304 & 30.1 & 10.1 \\
21 & 397 & 35.1 & 11.3 \\
21 & 500 & 37.6 & 13.3 \\
23 & 605 & 42 & 14.4 \\
$16^{*}$ & $683(160)$ & $46.5(30.1)$ & $14.7(5.3)$ \\
22 & 706 & 47.1 & 15 \\
28 & 784 & 49.3 & 15.9 \\
$8^{*}$ & $592(210)$ & $44.5(35)$ & $13.3(6.0)$ \\
21 & 655 & 45.5 & 14.4 \\
25 & 898 & 54.1 & 16.6 \\
\hline \hline
\end{tabular}

distance of $3.5 \mathrm{~m}$ from the extraction and acceleration system ${ }^{19}$ to stop the beam. Each block is made of pure copper $(38 \times 38$ $\times 25 \mathrm{~mm}$ ) and can be considered as an inertial dump. It is cooled down in the time interval between two consecutive pulses by the cooling system on the rear side of the blocks. The side facing the beam is coated with molybdenum disulfide $\left(\mathrm{MoS}_{2}\right)$ to perform IR analysis. This is done by a FLIR A655sc IR micro-bolometer camera which is triggered $5 \mathrm{~s}$ before the $\mathrm{HV}$ phase and acquires IR images for $1 \mathrm{~min}$, including the $10 \mathrm{~s}$ beam time. Thermocouples installed in 48 copper blocks of the calorimeter make it possible to calibrate the camera on an absolute scale. From the power deposition on the calorimeter surface, one can then determine a $2 \mathrm{D}$ profile of the beam current on the dump, as well as the beam size and uniformity for later use in the calculations (see Sec. III). A 2D beam power map (profile) at low $(300 \mathrm{~kW})$ and high $(900 \mathrm{~kW})$ power deposition $\left(\mathrm{W} / \mathrm{m}^{2}\right)$ is shown in Fig. 1. The profile is very similar in the two cases as, in the experiment, we have designed the beam parameters on purpose to achieve comparable profiles at different powers.

\section{B. Time trace of neutron emission measurements}

Neutron emission is measured by the calibrated ScionixEJ301A liquid scintillator used also in our previous experiment. ${ }^{12}$ The scintillator measures the neutron counting rate and was coupled to an active base H10580 Hamamatsu photomultiplier tube (PMT). The detector was installed in vicinity of one of the two inner walls of the ELISE facility, at a distance of approximately $2.8 \mathrm{~m}$ behind the beam dump and with same experimental arrangement described in Ref. 12. Signals coming from the detector were digitized by means of a 14 bit, $400 \mathrm{MS} / \mathrm{s}$ custom digitizer based on the Advanced Telecommunications Computing Architecture (ATCA) platform. ${ }^{20,21}$ A neutron low energy threshold of $1.15 \mathrm{MeV}$ was used in the measurements. Neutron/gamma-ray discrimination is based on standard long/short gate charge integration. We estimate the probability of a wrong neutron/gamma-ray event discrimination to be about $6 \%$. A neutron/gamma-ray ratio of about 3 was found experimentally.

\section{LOCAL MIXING MODEL BASED CALCULATIONS}

Neutron emission calculations are based on the LMM model of deuterium implantation in the dump, where diffusion or migration of deuterium is neglected once it is implanted. In more details, the flux of deuterium beam on the $i$ th row and $j$ th column block of the dump, at a depth $z$ and at a time $t$, $\Phi_{i, j}(z, t)$, can be calculated as

$$
\Phi_{i, j}(z, t)=\Phi_{i, j}(0, t)\left(1-\int_{0}^{z} p(x) d x\right) .
$$

Here $p(x)$ is the probability for the deposition of a deuteron at a depth $x$ and depends on the incident energy significantly. In the LMM, no other reactions but deposition has been assumed and the probability of deposition in depth has been normalised to one. The TRansport of Ion in Matter (TRIM) $\operatorname{code}^{22}$ has been applied to calculate the $p(x)$ for $27-60 \mathrm{keV}$ incident deuterons with a step of $0.5 \mathrm{keV}$. The dump density was set to $8.902 \mathrm{~g} / \mathrm{cm}^{3} . \Phi_{i, j}(0, t)$ is the incident deuterium flux. This is 

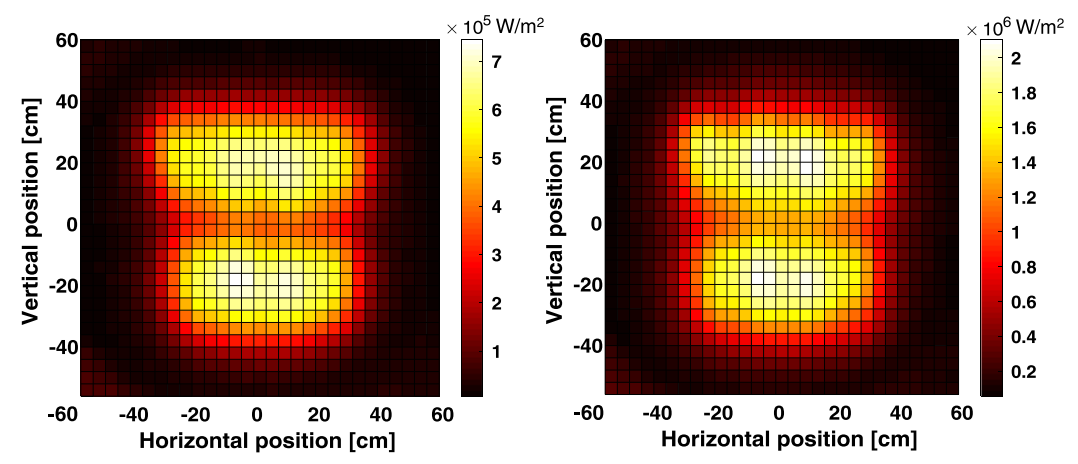

FIG. 1. Beam profile measured in the neutron emission experiment by the ELISE infra-red camera at low $(300 \mathrm{~kW}$, left) and high (900 kW, right) power deposition. in turn given by

$$
\Phi_{i, j}(0, t)=\frac{I_{i, j}}{\Delta S \cdot q},
$$

where $I_{i, j}$ is the current that reaches the $i$ th row and $j$ th column block of the dump, $\Delta S$ is the area of each block, and $q$ is the ion charge. $I_{i, j}$ is obtained by dividing the known power on each block by the acceleration voltage.

The time resolved neutron yield from the dump $Y(t)$ can be determined from $\Phi_{i, j}(z, t)$ as

$$
Y(t)=\sum_{i=1}^{30} \sum_{j=1}^{30} \int_{0}^{R} \Phi_{i, j}(z, t) n_{i, j}(z, t) \sigma(z, t) d z \times \Delta S .
$$

Here, $n_{i, j}(z, t)$ is the time dependent number of deuterons that get deposited per unit volume at a depth $z$, and $\sigma(z, t)$ is the $D(d, n)^{3}$ He neutron production cross section (see below).

Ion implantation of hydrogen isotopes into metals can yield high concentrations of atoms. ${ }^{23}$ The concentration here is defined as the percentage of deuterons to target atoms per unit volume. As the incident deuterons penetrate in the dump, the local deuteron concentration increases until a maximum is reached (saturation) and which depends on the target material. ${ }^{13}$ Based on the results of our previous experiment,,${ }^{9,12}$ we have used $20 \%$ as concentration at saturation. ${ }^{24}$

The cross section $\sigma(z, t)$ is related to the deuteron energy. The energies of deuterons at depth $z$ have been calculated by using the total stopping power data from the TRIM code so as

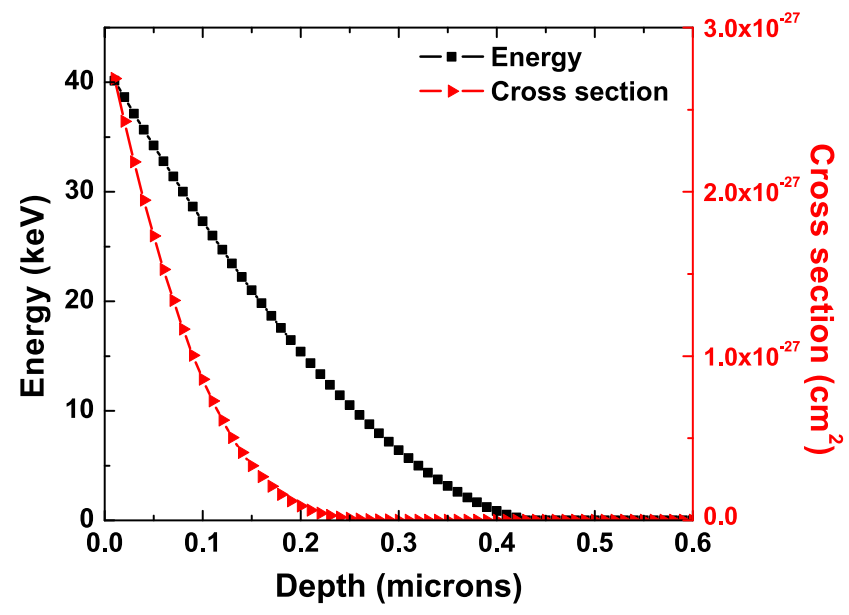

FIG. 2. Deuteron energy (left scale) and the $D(d, n)^{3} \mathrm{He}$ cross section (right scale) as a function of depth at $40 \mathrm{keV}$. to convert the energy dependent $D(d, n)^{3} \mathrm{He}$ cross section to a function that depends on the depth $z$. Figure 2 shows an example of the deuteron energy and the $D(d, n)^{3} \mathrm{He}$ cross section as a function of depth for $40 \mathrm{keV}$ incident deuterons. From the figure, we note that the effective layer from which neutrons are produced is only $0.5 \mu \mathrm{m}$ thick and that most neutrons are produced within the first $0.2 \mu \mathrm{m}$.

\section{RESULTS}

Before the dedicated experiment, a set of experiments with a 22-44 keV deuterium beam was performed from the beginning of the deuterium campaign so that we could assume that deuterium density reached saturation before our dedicated investigation. Figure 3 shows the LMM based calculation of the time trace of neutron emission together with the measurement in our dedicated experiment. The calculation is based on Eq. (3) and uses as input the measured diagnostic information on the beam (current, voltage, and profile). In general, the LMM based calculation reproduces the basic features of the emission, i.e., the step ladder increase of the neutron yield as the beam power on the target is increased. In order to compare simulations and measurements in a more quantitative way, the conversion coefficient $k$ from $Y(t)$ (neutron/s from the target) to the detector counting rate must be determined. As $k$ depends only on the neutron transport from the dump to the detector, it can be empirically evaluated by the ratio between the simulation and measurements at one experimental point where the

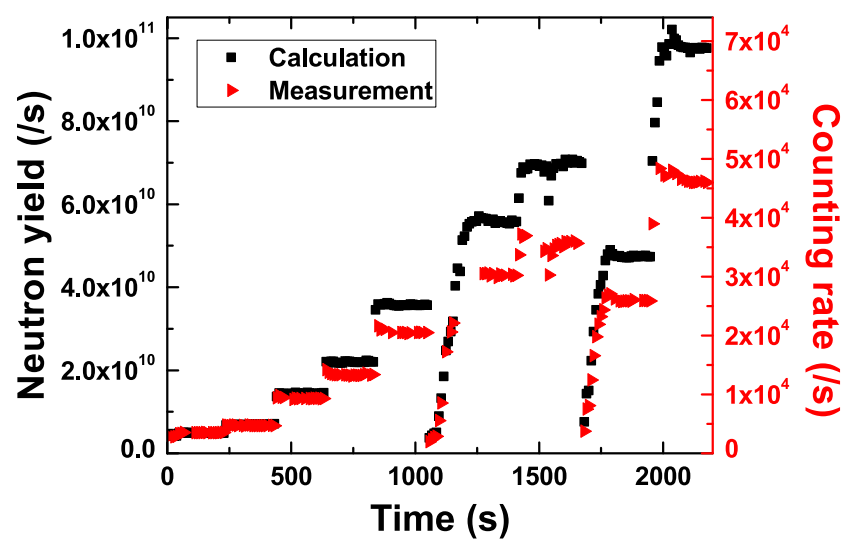

FIG. 3. Calculated neutron emission from the dump as a function of time (left axis) compared to the measured counting rate (right axis). 


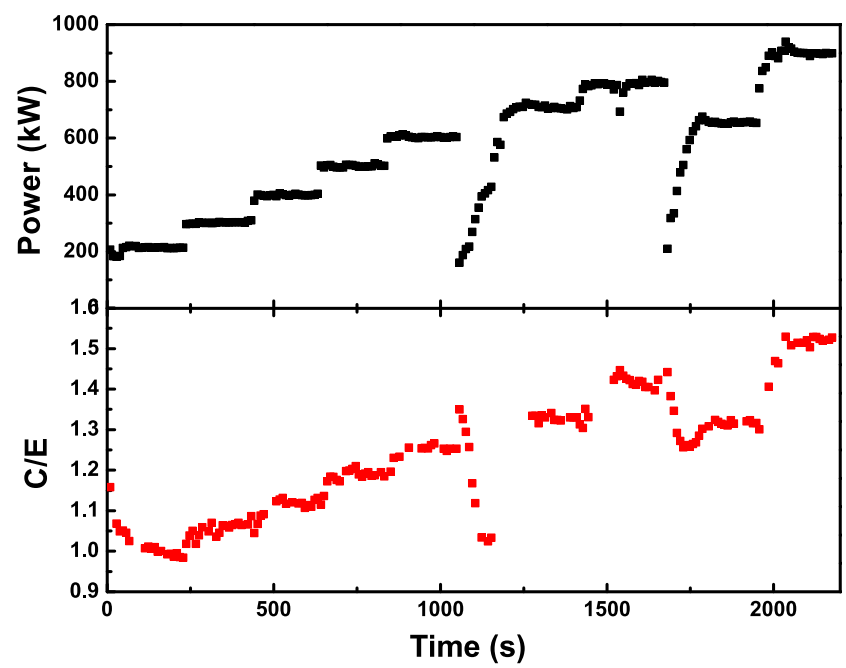

FIG. 4. (top) Beam power impinging on the dump as a function of time (bottom) Ratio between the calculated and measured neutron yield as a function of time.

LMM based calculation is assumed to be correct, and data at $200 \mathrm{~kW}$ on the dump between $\mathrm{t}=114 \mathrm{~s}$ and $\mathrm{t}=209 \mathrm{~s}$ have been used to calculate $k$.

Using this value, we can then compare measurements and calculations on a quantitative ground, albeit on a relative scale, as shown in Fig. 4. If the LMM model held exactly at any power on the dump, we would expect a ratio between the calculated and experimental neutron rate $C / E=1$, but this contradicts the experimental data. Instead, we observe that the model calculates systematically more neutrons than found experimentally, i.e., $C / E>1$. A graph of $C / E$ as a function of the power on the dump (Fig. 5) shows a linear correlation between the two parameters, but for a few outliers. These correspond to the first few pulses of each experimental day when, presumably, a full saturation of the deuterium concentration in the dump was not yet reached. A linear fit to the data of Fig. 5 yields $C / E=(7.1 \pm 0.1) \times 10^{-4} \times$ Power $+(0.841$ $\pm 0.008)$. The $\mathrm{C} / \mathrm{E}$ values obtained by the experimental fit in this paper agree within $5 \%$ with those found in our previous study $^{15}$ in the lower power range where the two experiments overlap.

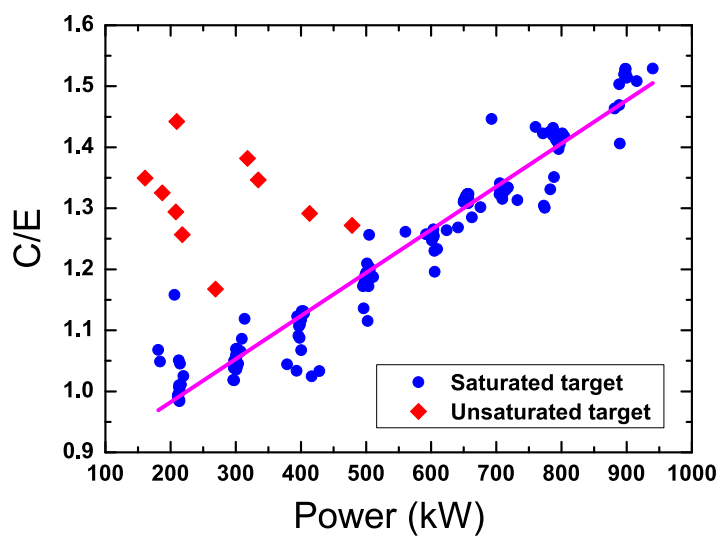

FIG. 5. Ratio $C / E$ between the calculated and measured neutron emission as a function of the power on the dump.

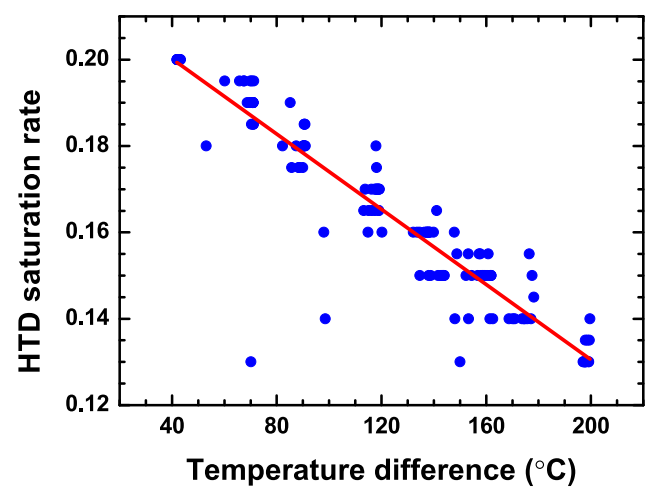

FIG. 6. Temperature dependent saturation rate as a function of the temperature difference after and before the beam irradiation.

\section{DISCUSSION}

LMM based calculations require three measured quantities as input, i.e., the beam profile, the current on the dump, and the beam voltage. Each of them has an estimated uncertainty of 5\%, which yields to an uncertainty in the calculation and is reflected in the fluctuation of the points at each constant power level in Fig. 5. Although this is certainly a visible effect, its magnitude is lower than the systematic trend of the deficit observed in the same figure and we judge it to have a negligible impact on our conclusion on the discrepancy between LMM based calculation and data.

In order to understand the results of Fig. 5, we can make the hypothesis that the dominant effect is a reduction of the deuterium concentration at saturation as a function of temperature. Qualitatively, one can expect temperature to promote the diffusion of deuterium in the dump and we here speculate that this is manifested in the different concentrations reached at saturation as a function of temperature. The IR diagnostics installed at ELISE determines the difference $\Delta T$ between the average temperature of each individual block of the beam dump before and after irradiation. ${ }^{11}$ By defining the hypothetical temperature dependent saturation rate (HTDSR) as the concentration at saturation required to exactly match the experimental data at each temperature, we can use the results of the experiment to find HTDSR as a function of $\Delta T$ averaged over the dump surface (Fig. 6). As for Fig. 3, we have assumed that the concentration at saturation is $20 \%$ at a power of $200 \mathrm{~kW}$. Data show a linear decrease of HTDSR versus $\Delta T$ which is described as HTDSR $=-4.35 \times 10^{-4} \times \Delta T+0.218$. This formula can be used as an empirical correction for neutron emission calculations at SPIDER.

\section{CONCLUSIONS}

A dedicated experiment on beam-target neutron emission has been performed at the ELISE neutral beam test facility to study the discrepancy between measurements and calculations in a power density range approaching that of SPIDER. The time trace of the neutron emission has been monitored by a calibrated neutron detector and the local mixing model has been applied for predictions. The ratio between calculations and measurements $(\mathrm{C} / \mathrm{E})$ on a relative scale has been found 
to exceed unity beyond the observed fluctuation level of the data points at constant power and to scale linearly with the beam power in the range $200 \mathrm{~kW}-950 \mathrm{~kW}$. Assuming this can be explained by a temperature dependent saturation rate of deuterons in the target, an empirical correction to neutron emission calculations for applications at SPIDER has been derived.

\section{ACKNOWLEDGMENTS}

The work leading to this publication was funded partially by the Fusion for Energy under the Contract No. F4ERFXPMS A-WP-2017. The views and expression expressed herein do not necessarily reflect those of the Fusion for Energy and ITER organizations.

${ }^{1}$ V. Toigo, D. Boilson, T. Bonicelli, R. Piovan, M. Hanada, A. Chakraborty, G. Agarici, V. Antoni, U. Baruah, M. Bigi et al., Nucl. Fusion 55, 083025 (2015).

${ }^{2}$ V. Toigo, R. Piovan, S. Dal Bello, E. Gaio, A. Luchetta, R. Pasqualotto, P. Zaccaria, M. Bigi, G. Chitarin, D. Marcuzzi et al., New J. Phys. 19, 085004 (2017).

${ }^{3}$ R. Pasqualotto, M. Agostini, M. Barbisan, M. Bernardi, M. Brombin, R. Cavazzana, G. Croci, M. D. Palma, R. Delogu, G. Gorini et al., AIP Conf. Proc. 1869, 030020 (2017).

${ }^{4}$ F. Murtas, G. Croci, G. Claps, M. Cavenago, M. Dalla Palma, G. Gervasini, G. Grosso, R. Pasqualotto, E. P. Cippo, M. Rebai et al., in 2011 IEEE Nuclear Science Symposium and Medical Imaging Conference (NSS/MIC) (IEEE, 2011), pp. 405-408.

${ }^{5}$ G. Croci, M. Rebai, G. Claps, M. Cavenago, M. Dalla Palma, G. Gervasini, G. Grosso, F. Murtas, R. Pasqualotto, E. P. Cippo et al., J. Instrum. 7, C03010 (2012).

${ }^{6}$ A. Muraro, G. Croci, G. Albani, C. Cazzaniga, G. Claps, M. Cavenago, G. Grosso, M. Dalla Palma, M. Fincato, F. Murtas et al., Fusion Eng. Des. 96, 311 (2015).

${ }^{7}$ A. Muraro, G. Croci, G. Albani, G. Claps, M. Cavenago, C. Cazzaniga, M. Dalla Palma, G. Grosso, F. Murtas, R. Pasqualotto et al., Nucl. Instrum. Methods Phys. Res., Sect. A 813, 147 (2016).
${ }^{8}$ M. Rebai, M. Cavenago, G. Croci, M. Dalla Palma, G. Gervasini, F. Ghezzi, G. Grosso, F. Murtas, R. Pasqualotto, E. Perelli Cippo et al., Rev. Sci. Instrum. 83, 02B721 (2012).

${ }^{9}$ M. Rebai, Ph.D. thesis, Università degli Studi di Milano-Bicocca, 2012, http://hdl.handle.net/10281/28449.

${ }^{10}$ M. Rebai, G. Croci, G. Grosso, A. Muraro, E. P. Cippo, M. Tardocchi, M. Dalla Palma, R. Pasqualotto, M. Tollin, F. Murtas et al., J. Instrum. 12, C01007 (2017).

${ }^{11}$ R. Nocentini, F. Bonomo, A. Pimazzoni, U. Fantz, P. Franzen, M. Fröschle, B. Heinemann, R. Pasqualotto, R. Riedl, B. Ruf et al., AIP Conf. Proc. 1655, 060006 (2015).

${ }^{12}$ X. Xufei, M. Nocente, F. Bonomo, P. Franzen, M. Fröschle, G. Grosso, F. Grünauer, R. Pasqualotto, M. Tardocchi, T. Fan et al., Rev. Sci. Instrum. 85, 11 D864 (2014).

${ }^{13}$ B. L. Doyle, D. K. Brice, and W. R. Wampler, Radiat. Eff. 57(3), 81 (1981).

${ }^{14}$ D. Brice, B. Doyle, and W. Wampler, J. Nucl. Mater. 111, 598 (1982).

${ }^{15}$ M. Nocente, S. Feng, D. Wünderlich, F. Bonomo, G. Croci, U. Fantz, B. Heinemann, W. Kraus, I. Mario, R. Pasqualotto et al., Fusion Eng. Des. 123, 843 (2017).

${ }^{16}$ P. Franzen, U. Fantz, D. Wünderlich, B. Heinemann, R. Riedl, W. Kraus, M. Fröschle, B. Ruf, R. Nocentini, NNBI Team et al., Nucl. Fusion 55 , 053005 (2015).

${ }^{17}$ D. Wünderlich, U. Fantz, B. Heinemann, W. Kraus, R. Riedl, C. Wimmer, NNBI Team et al., Nucl. Fusion 56, 106004 (2016).

${ }^{18}$ A. Pimazzoni, Ph.D. thesis, Università degli Studi di Padova, 2014, http://tesi.cab.unipd.it/46495/.

${ }^{19}$ R. Nocentini, U. Fantz, P. Franzen, M. Froeschle, B. Heinemann, R. Riedl, B. Ruf, D. Wuenderlich et al., Fusion Eng. Des. 88, 913 (2013).

${ }^{20}$ M. Nocente, M. Tardocchi, I. Chugunov, R. Pereira, T. Edlington, A. Fernandes, D. Gin, G. Grosso, V. Kiptily, A. Murari et al., Rev. Sci. Instrum. 81, 10D321 (2010).

${ }^{21}$ M. Nocente, M. Tardocchi, A. Olariu, S. Olariu, R. Pereira, I. Chugunov, A. Fernandes, D. Gin, G. Grosso, V. Kiptily et al., IEEE Trans. Nucl. Sci. 60, 1408 (2013).

${ }^{22}$ J. F. Ziegler, M. D. Ziegler, and J. P. Biersack, Nucl. Instrum. Methods Phys, Res., Sect. B 268, 1818 (2010).

${ }^{23}$ W. Möller, Nucl. Instrum. Methods Phys. Res. 209, 773 (1983).

${ }^{24}$ J. Kim, Nucl. Technol. 44, 315 (1979). 